\title{
Low-Energy Adaptive Cooperative Diversity Applied to Wireless Sensor Networks
}

\author{
Marcelo P. Sousa, Ajey Kumar, Marcelo S. Alencar and Waslon T. A. Lopes
}

\begin{abstract}
The performance of wireless sensor networks (WSNs) can be significantly improved using adaptive cooperative diversity (SCA) technique, which is basically a cross-layer design that combines truncated ARQ at the link layer and cooperative diversity at the physical layer. LEACH is a protocol for wireless sensor networks that utilizes randomized rotation of local clusterheads to evenly distribute the energy load among the sensors in the network. This paper proposes a combination of the adaptive cooperative diversity and LEACH techniques for wireless sensor networks. Simulation results show that the proposed scheme (SCA with LEACH) has satisfatory lifetime and packet loss rate performances.
\end{abstract}

Keywords-WSN, cooperative diversity, truncated ARQ, crosslayer design, SCA, LEACH, SCA with LEACH.

\section{INTRODUCTION}

Recent advances in micro-electro-mechanical systems (MEMS) technology, wireless communications, and digital eletronics have enabled the development of low-cost, low-power, multifunctional sensor nodes. A wireless sensor network (WSN) [1] consists of sensor nodes deployed over a geographical area for monitoring physical phenomena including temperature, humidity, vibrations, seismic events. Typically, a sensor node is a tiny device that has three basic components: a sensing subsystem for data acquisition from the physical surrounding environment, a processing subsystem for local data processing and storage, and a wireless communication subsystem for data transmission. In addition, a power source supplies the energy needed by the device to perform the programmed task. This power source often consists of a battery with a limited energy budget. In addition, it could be inconvenient to recharge the battery, because nodes may be deployed in a hostile or unpractical environment. On the other hand, the sensor network should have a lifetime long enough to fulfill the application requirements [2]. Several researches have been carried out in industry/academia for energy conservation in WSNs.

Various methods have been provided which intend to increase the lifetime of WSNs. Cluster based protocols are one of the successful methods for energy saving. Heinzelman et al developed LEACH (Low-Energy Adaptive Clustering Hierarchy) [3], a clustering-based protocol that minimizes energy dissipation in sensor networks. The use of clusters for transmitting data to the sink node leverages the advantages

Marcelo P. Sousa, Ajey Kumar, Marcelo S. Alencar, Instituto de Estudos Avançados em Comunicações, Universidade Federal de Campina Grande, Brasil. Waslon T. A. Lopes, ÁREA1FTE, Salvador, Brasil. E-mails: \{marporsou, ajeykumar, malencar\}@iecom.org.br, waslon.lopes@area1fte.edu.br. Este trabalho foi apoiado pelo $\mathrm{CNPq}$ e pelo Iecom of small transmit distances for most nodes, requiring only a few nodes to transmit far distances to the sink node. However, LEACH outperforms classical clustering algorithms by using adaptive clusters and rotating cluster-heads, allowing the energy requirements of the system to be distributed among all the sensors. In addition, LEACH is able to perform local computation in each cluster to reduce the amount of data that must be transmitted to the sink node. This achieves a large reduction in the energy dissipation, as computation is much cheaper than communication [3].

In WSN channels, the fading caused by multipath, can significantly degrade the performance of the communication system. Diversity techniques [4], [5], [6] can improve the performance of those systems, since replicas of the transmitted signals are provided to the receiver sink node. Time diversity, frequency diversity, spatial diversity and modulation diversity are examples of typical diversity techniques.

Unfortunately, the application of diversity techniques by the use of multiple antennas, could be impractical in a wireless sensor network, because of the size of the sensor nodes and the energy constraints present in the network. In order to overcome this limitation, a new form of time-spatial diversity has been proposed, whereby diversity gains are achieved via the cooperation of the nodes. Lin Dai et al proposed the SCA (Selective Cooperative diversity with ARQ) scheme, in which adaptive cooperative diversity gain can be achieved and error propagation is therefore avoided.

The authors in [7] introduced Space-Time Block Coding (STBC), a paradigm for communication over Rayleigh fading channels using multiple transmit antennas. They extended the proposal presented in [8], in which the author designed a diversity scheme for two transmit and two receive antennas, at most. Data is encoded using STBC and the encoded data is split into $n$ streams which are simultaneously transmitted using $n$ transmit antennas. The received signal at each receive antenna is a linear superposition of the $n$ transmitted signals perturbed by noise. Maximum likelihood decoding is achieved in a simple way through decoupling of the signals transmitted from different antennas rather than joint detection. STBC provides full diversity and very low complexity encoding and decoding [9].

Automatic Repeat Request (ARQ) protocol at the link layer is an effective means to overcome the channel fading, in which Cyclic Redundancy Check (CRC) is used for error check and retransmissions are requested if the packet is received erroneously [10], [11]. In practice, the maximum number of retransmissions is limited so as to minimize the delay and buffer size, and such variant ARQ is called truncated ARQ 
protocol [10].

In this paper the authors propose a system, SCA with LEACH, that integrates the SCA cross-layer design with the LEACH protocol, for wireless sensor networks. STBC is the adopted diversity scheme. The lifetime performance and packet loss rate of the system are evaluated and compared with the LEACH with truncated ARQ system. Furthermore, two important thresholds are provided, that relate the maximum number of retransmissions and the lifetime performance.

The remaining of the paper is organized as follows: Sections II and III provide a brief description of the LEACH protocol and of the SCA scheme, respectively. The proposed system, the channel model used and the STBC operation are described in Section IV. Simulation parameters and results are discussed in Section $\mathrm{V}$ and the paper is concluded in Section VI.

\section{THE LEACH PROTOCOL}

LEACH is a self-organizing, adaptive clustering protocol that uses randomization to distribute the energy load evenly among the sensors in the network. In LEACH the nodes organize themselves into local clusters, with one node acting as the cluster-head. In addition, LEACH performs local data fusion to compress the amount of data being sent from the clusters to the sink node, further reducing energy dissipation and enhancing system lifetime [3].

The LEACH protocol provides a conception of round, and contains two states: the cluster set-up state and the steady state. In the cluster set-up state it forms a cluster in self-adaptive mode. In the steady state it transfers data [12].

Initially, when clusters are being created, each node decides whether to become a cluster-head for the current round. This decision is based on the suggested percentage of cluster-heads for the network (determined a priori) and the number of times the node has been a cluster-head so far. This decision is made by node $n$ choosing a random number between 0 and 1 . If the number is less than a threshold $T(n)$ the node becomes a cluster-head for the current round. The threshold is set as [3]

$$
T(n)= \begin{cases}\frac{P}{1-P \times\left(r \bmod \frac{1}{P}\right)} & \text { if } n \in G \\ 0 & \text { otherwise, }\end{cases}
$$

in which $P$ is the desired percentage of cluster-heads, $r$ is the current round, and $G$ is the set of nodes that have not been cluster-heads in the last $\frac{1}{P}$ rounds. Using this threshold, each node will be a cluster-head at some point within $\frac{1}{P}$ rounds. Fig. 1 shows the cluster formation in the network, in which each cluster is represented by a Voronoi region.

Once the clusters are created and a TDMA schedule is fixed data transmission can begin. This is the steady-state operation to LEACH networks. Then, the next round begins with each node determining if it should be a cluster-head for this round and advertising this information.

\section{SCA}

Selective Cooperative diversity with ARQ (SCA) is a crosslayer design which combines truncated ARQ at the data link

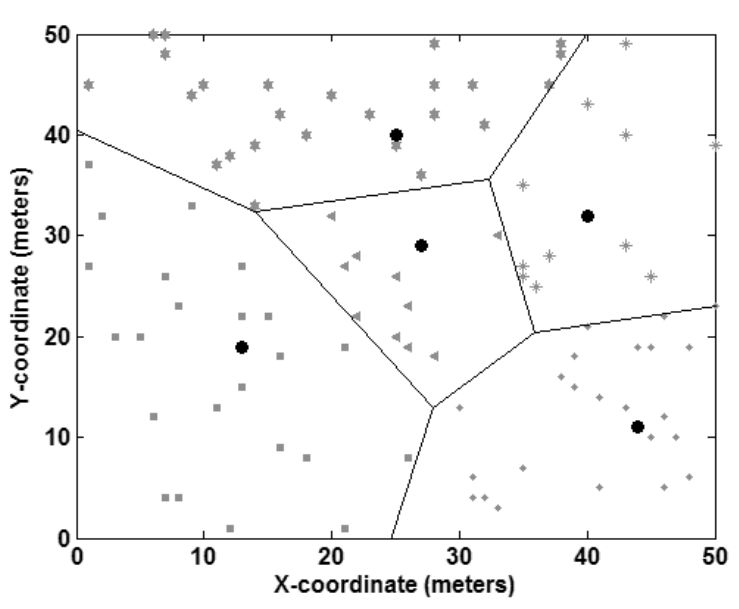

Fig. 1. Clusters as Voronoi regions.

layer and cooperative diversity at the physical layer [13]. With this combination, adaptive cooperative diversity gain can be reached. Besides, the channel quality is significantly improved in the retransmissions by using relays so that substantial throughput gains can be obtained [13].

SCA uses orthogonal relay transmission to achieve diversity gain. Each node has one or several partners. The node and its partner(s) are responsible for transmitting not only their own information, but also the information of their partner(s). Therefore, a virtual antenna array is obtained with the use of the relays' antennas without complicated signal design or adding more antennas to the nodes [13].

In the SCA scheme, error propagation can be avoided, since only the relay candidates who correctly detect the packet are selected to be relay nodes. Besides, node cooperation is adopted only when the receiver fails to detect the packet correctly. Therefore, the cooperative diversity is employed in a selective and an adaptive manner.

\section{SCA WITH LEACH}

This paper assumes a wireless sensor network with $K$ nodes and each node is equipped with one antenna. Among the $K$ nodes, $Q$ nodes are the cluster-heads of the correspondent round selected by the LEACH algorithm, $Q-1$ idle clusterheads are assumed to be available as the possible relays for the source cluster-head during the packet transmission. The source cluster-head transmits a data packet with a $C$-bit CRC attached and the sink node detects the CRC. Then, an acknowledgement that is either positive (ACK) or negative (NACK) is sent back to the source cluster-head, from the sink node. At the same time, all the $Q-1$ relay candidates check the CRC and the ones who get positive results are selected to be relay cluster-heads. If the packet is correctly detected by the sink node (with ACK feedback), the source cluster-head continues to transmit a new data packet and the above process is repeated. Otherwise, retransmission will start. Both the source and the relay cluster-heads will jointly retransmit the packet by utilizing a suitable orthogonal STBC. The retransmission continues until the packet is successfully 
delivered, or the number of retransmissions exceeds $N_{r}^{\max }$, which is a preset parameter indicating the maximum number of retransmissions allowed per packet.

\section{A. Channel Model}

The considered channel model was introduced in [13] and is presented in Fig. 2. The communication between the source cluster-head and the sink node is assumed to be over a flat Rayleigh fading channel and facilitated by $v$ relay clusterheads, which are selected from $Q-1$ relay candidates. In addition, perfect channel knowledge is assumed to be available at the receiver side only, through the use of training sequences.

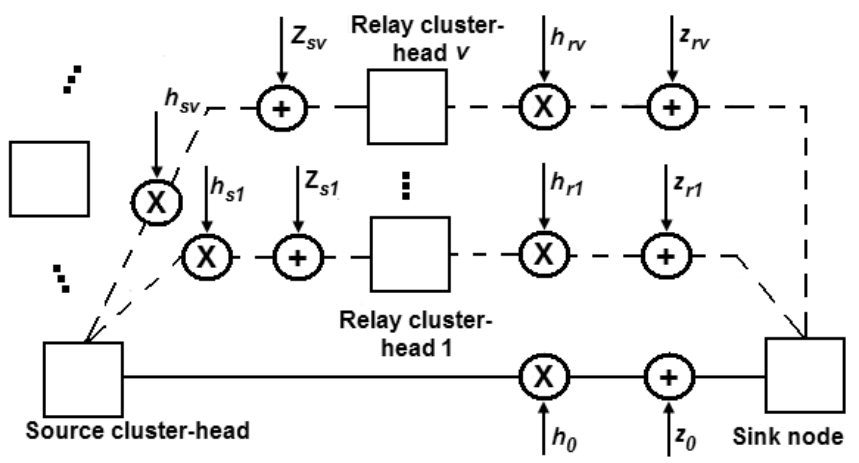

Fig. 2. Channel model scheme.

At time slots $t_{0}+1, \ldots, t_{0}+\varsigma$, the source cluster-head sends a packet $x_{t_{0}+1}^{s}, \ldots, x_{t_{0}+\varsigma}^{s}$ with transmission power $P_{t}$ per symbol, in which $x_{t_{0}+i}^{s}$ is an $M$-QAM modulated symbol and $\varsigma=L / b$ is the number of symbols per packet with a total packet length of $L$ bits and a modulation level of $b=$ $\log _{2} M$ bits. The signal received by the sink node at time slot $t_{0}+i, i=1, \ldots, \varsigma$, is then given by

$$
y_{t_{0}+i}^{d}=h_{0} x_{t_{0}+i}^{s}+z_{0}
$$

in which the channel gain $h_{0}$ is assumed to be a complex Gaussian random variable with zero-mean and variance $\sigma_{0}^{2}$, and $\sigma_{0}^{2}$ accounts for the effect of large-scale path loss and shodowing. Also, $z_{0}$ represents the additive white Gaussian noise with zero-mean and variance $N_{0}$. At the $j$-th relay candidate, $j=1, \ldots, Q-1$, the received signal is given by

$$
y_{t_{0}+1}^{r_{j}}=h_{s j} x_{t_{0}+i}^{s}+z_{s j},
$$

in which the noise $z_{s j}$ and the fading coefficient $h_{s j}$ are complex Gaussian random variables with zero-mean and variance $N_{0}$ and $\sigma_{s_{j}}^{2}$, respectively, $j=1, \ldots, Q-1$. In the following, the packet $x_{t_{0}+1}^{s}, \ldots, x_{t_{0}+\varsigma}^{s}$ is directly transmitted.

If the sink node fails to detect the packet correctly retransmission starts at time slot $t_{L}+1$. Let $N_{r}$ be the number of retransmissions, $1 \leq N_{r} \leq N_{r}^{\max }$. The received signal at the sink node at time slot $t_{L}+i$ is given by

$$
y_{t_{L}+i}^{d}=h_{0} x_{t_{L}+i}^{s}+z_{0}+\sum_{j=1}^{v} h_{r j} x_{t_{L}+i}^{r_{j}}+z_{r j},
$$

for $i=1, \ldots, N_{r} \varsigma / R$, in which $R$ is the rate of the STBC. The symbols $x_{t_{L}+i}^{s}$ and $x_{t_{L}+i}^{r_{j}}$ are the STBC transmitted by the source cluster-head and the $j$-th relay cluster-head with the transmission power $P_{t} /(v+1)$, respectively. The additive noise $z_{r j}$ and the fading coefficient $h_{r j}$ are complex Gaussian random variables with zero-mean and variance $N_{0}$ and $\sigma_{r_{j}}^{2}$, respectively, $j=1, \ldots, v$.

\section{B. Space-Time Block Codes}

The Alamouti scheme is the first STBC to provide full transmit diversity for systems with two transmit antennas [14].

Assume a system with two transmitter cluster-heads and one receiver sink node, both with just one antenna employing the Alamouti code and using the QPSK modulation. First, one of the the cluster-heads picks the symbol $s_{1}$ from the constellation and the other picks the symbol $s_{2}$. Then, they transmit these respective symbols at time one and, at time two, they transmit $-s_{2}^{*}$ and $s_{1}^{*}$, respectively. Therefore, the transmitted codeword is

$$
\mathcal{C}=\left(\begin{array}{cc}
s_{1} & s_{2} \\
-s_{2}^{*} & s_{1}^{*}
\end{array}\right) .
$$

Consider that the path gains from transmitter cluster-heads one and two to the sink node are $\alpha_{1}$ and $\alpha_{2}$, respectively. Then, the decoder receives signals $r_{1}$ and $r_{2}$ at times one and two, respectively, such that

$$
\left\{\begin{array}{l}
r_{1}=\alpha_{1} s_{1}+\alpha_{2} s_{2}+\eta_{1} \\
r_{2}=-\alpha_{1} s_{2}^{*}+\alpha_{2} s_{1}^{*}+\eta_{2} .
\end{array}\right.
$$

For a coherent detection scheme the sink node knows the channel path gains $\alpha_{1}$ and $\alpha_{2}$, the maximum-likelihood detection amounts to minimizing the decision metric

$$
\left|r_{1}-\alpha_{1} s_{1}-\alpha_{2} s_{2}\right|^{2}+\left|r_{2}-\alpha_{1} s_{2}^{*}-\alpha_{2} s_{1}^{*}\right|^{2},
$$

over all possible values of $s_{1}$ and $s_{2}$. Such decoding scheme requires a full search over all possible pairs $\left(s_{1}, s_{2}\right)$ and in general its complexity grows exponentially with the number of transmitter cluster-heads. Expanding the cost function (7), one can ignore the common term $\left|r_{1}\right|^{2}+\left|r_{2}\right|^{2}$. Then, the metric (7) decomposes into two parts, one of which [9]

$$
\left|s_{1}\right|^{2} \sum_{n=1}^{2}\left|\alpha_{n}\right|^{2}-\left[r_{1} \alpha_{1}^{*} s_{1}^{*}+r_{1}^{*} \alpha_{1} s_{1}+r_{2} \alpha_{2}^{*} s_{1}+r_{2}^{*} \alpha_{2} s_{1}^{*}\right] \text {, }
$$

is a function of $s_{1}$ only, and the other one

$$
\left|s_{2}\right|^{2} \sum_{n=1}^{2}\left|\alpha_{n}\right|^{2}-\left[r_{1} \alpha_{2}^{*} s_{2}^{*}+r_{1}^{*} \alpha_{2} s_{2}-r_{2} \alpha_{1}^{*} s_{2}-r_{2}^{*} \alpha_{1} s_{2}^{*}\right],
$$

depends on $s_{2}$. Therefore, instead of minimizing the cost function (7) over all possible values of $\left(s_{1}, s_{2}\right)$, one can simultaneously minimize the cost functions (8) and (9) over all possible values of $s_{1}$ and $s_{2}$, respectively. As a result the decoding complexity of the code increases linearly, instead of exponentially, by the number of transmitter clusterheads. In addition, since the constellation symbols have equal energy, as a QPSK scheme, the terms $\left|s_{1}\right|^{2} \sum_{n=1}^{2}\left|\alpha_{n}\right|^{2}$ and $\left|s_{2}\right|^{2} \sum_{n=1}^{2}\left|\alpha_{n}\right|^{2}$ can be ignored. In fact, the sink node should minimize

$$
\left|s_{1}-r_{1} \alpha_{1}^{*}-r_{2}^{*} \alpha_{2}\right|^{2}
$$


to decode $s_{1}$ and minimize

$$
\left|s_{2}-r_{1} \alpha_{2}^{*}+r_{2}^{*} \alpha_{1}\right|^{2}
$$

to decode $s_{2}$. Therefore, the decoding consists of first calculating

$$
\left\{\begin{array}{c}
\tilde{s}_{1}=r_{1} \alpha_{1}^{*}+r_{2}^{*} \alpha_{2} \\
\tilde{s}_{2}=r_{1} \alpha_{2}^{*}-r_{2}^{*} \alpha_{1}
\end{array}\right.
$$

Then, to decode $s_{1}$ the sink node finds the closest symbol to $\tilde{s}_{1}$ in the constellation. Similarly, the decoding of $s_{2}$ consists of finding the closest symbol to $\tilde{s}_{2}$ in the constellation [9].

The Alamouti scheme achieves the full diversity with a very simple maximum-likelihood decoding algorithm. The key feature of the scheme is orthogonality between the sequences generated by two transmitters. This scheme was generalized to an arbitrary number of transmitters by applying the theory of orthogonal designs. The generalized schemes are referred to as space-time block codes. The STBC's can achieve the full transmit diversity specified by the number of the transmitters, while allowing a very simple maximum-likelihood decoding algorithm, based only on linear processing of the received signals [14].

In this paper, cooperative diversity uses STBCs with two or three transmitter cluster-heads. For the former case, Alamouti coding is employed and the transmitted codeword is given by (5). For the last case, the STBC codeword is given by

$$
\mathcal{C}=\left(\begin{array}{cccc}
s_{1} & s_{2}^{*} & s_{3}^{*} & 0 \\
-s_{2} & s_{1}^{*} & 0 & -s_{3}^{*} \\
-s_{3} & 0 & s_{1}^{*} & s_{2}^{*}
\end{array}\right) .
$$

\section{Simulation Parameters And Results}

It is assumed that each node has an initial energy of $3 \mathrm{~mJ}$. The dissipation radio model used for the simulations was proposed in [3]. The radio dissipates $E_{\text {elec }}=50 \mathrm{~nJ} / \mathrm{bit}$ to run the transmitter or receiver circuitry and $\epsilon_{\mathrm{amp}}=100 \mathrm{pJ} / \mathrm{bit} / \mathrm{m}^{2}$ for the transmit amplifier to achieve an acceptable $\frac{E_{b}}{N_{0}}$ (the loss of energy in the transmission is assumed to depend on $r^{2}$ ). Thus, to transmit a $k$-bit message a distance $d$ using the radio model, the radio spends [3]

$$
E_{T x}(k, d)=E_{\text {elec }} \times k+\epsilon_{\mathrm{amp}} \times k \times d^{2}
$$

and to receive this message, the radio spends:

$$
E_{R x}(k)=E_{\text {elec }} \times k .
$$

The sensor network is formed by 100 nodes, with five clusters, each with one different cluster-head per round. Then, in (1), $P=0.05$ and for at last 20 rounds each node will be a cluster-head at some point. The nodes are deployed randomly in an area of $50 \times 50$ meters, as in Fig. 1. The sink node is located at the coordinates $x=25$ and $y=150$ meters.

Heinzelman et al did not mention if they used any ARQ scheme for the original LEACH protocol. But in order to evaluate the performance of the proposed system (SCA with LEACH) it is wise to compare it with LEACH with Truncated ARQ, instead of LEACH alone. This combination of the LEACH with Truncated ARQ consists of a system based in LEACH operation, but the Truncated ARQ is specified to be used as a link layer protocol. Then, retransmissions are alowed, but in a direct manner, without the use of diversity techniques. This system appends the truncated ARQ scheme for the original proposed LEACH protocol. The maximum number of retransmissions can change, based on the design needs.

Both systems use a truncated ARQ scheme. A CRC with $C=16$ bits is assumed with a cyclic generator polynomial of $\mathrm{G}_{\mathrm{CRC} 16}(D)=D^{16}+D^{12}+D^{5}+1$. The packet length $L$ is 120 bits and QPSK is the modulation scheme adopted. All the algorithms used for the simulations were developed using Matlab 7.

Four different propagation environments were used for the simulations, according to the random SNR distribution range of the propagation paths. When the source cluster-head transmits the signal, it travels through five different paths (four relay candidates and one sink node). The first scenario comprehends the following SNR range: $\left[\begin{array}{lllll}4 & 8 & 12 & 16 & 20\end{array}\right]$ $\mathrm{dB}$. These values are atributed randomly to each path in each round. The other SNR range scenarios, are shown in Table I. It is expected that the best performances can be reached as long as the last scenarios become the transmission option adopted for the simulation, because it is more probable the choose

\begin{tabular}{|c|c|c|c|c|c|}
\hline Scenario & \multicolumn{5}{|c|}{ SNR range (dB) } \\
\hline One & {$[4$} & 8 & 12 & 16 & $20]$ \\
\hline Two & {$[5$} & 10 & 15 & 20 & $25]$ \\
\hline Three & {$[6$} & 12 & 18 & 24 & $30]$ \\
\hline Four & {$[7$} & 14 & 21 & 28 & 35] \\
\hline
\end{tabular}
of higher SNR values and so, they have better propagation conditions than the first.

TABLE I

SNR RANGE SCENARIOS.

For comparing the Bit Error Rate (BER) between the schemes with and without diversity, Fig. 3 ilustrates a BER curve. The results are very similar to those found in [9]. The best performance is for the STBC scheme, with three transmitters and one receiver, followed by the Alamouti scheme, with two transmitters and one receiver. They are used for the diversity transmission stage. The poor performance corresponds to the QPSK scheme, which is used for the direct transmission stage (without diversity).

Network lifetime is the time span from the deployment to the instant when the network is considered nonfunctional. When a network should be considered nonfunctional is, however, application-specific. It can be, for example, the instant when the first sensor dies, a percentage of sensors die, the network partitions, or the loss of coverage occurs [15]. In this paper, the network lifetime measurement is based on the amount of rounds (time steps) that the first or last sensor node dies.

Figs. 4 and 5 show an overall behavior of the system lifetime performance for the sensor network as a function of the four scenarios considered in Table I. For better channel conditions (scenarios three and four), the lifetime is extended, since less errors occurs and less retransmissions are needed. Comparing Figs. 4 and 5, can be observed that SCA with LEACH presents 
better results than the LEACH with truncated ARQ system.

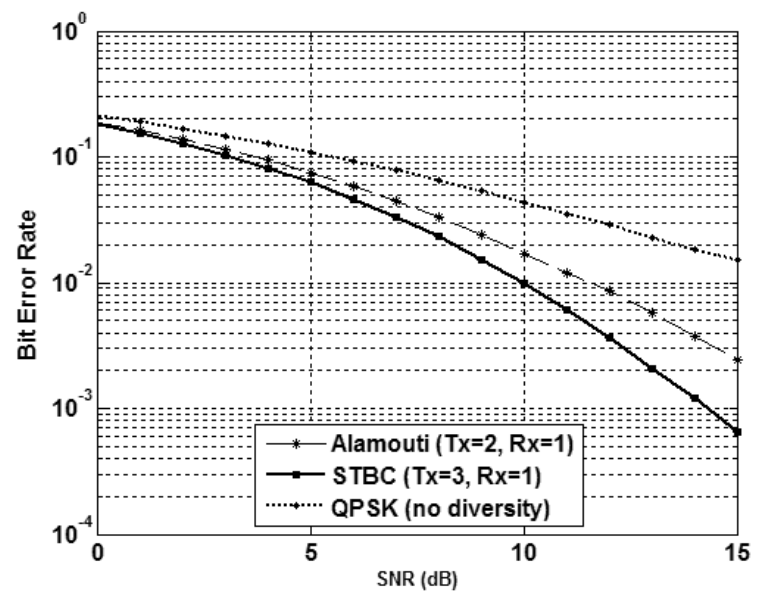

Fig. 3. Bit Error Rate as a function of the SNR.

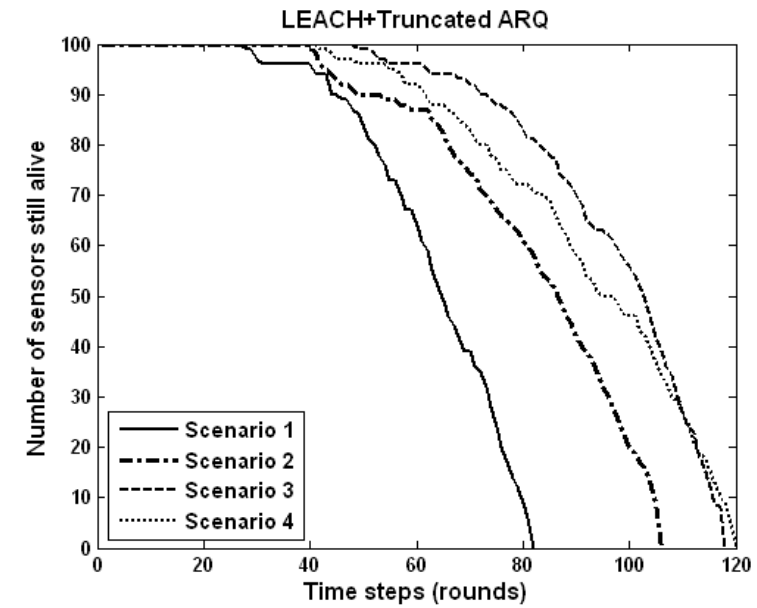

Fig. 4. System lifetime for LEACH with truncated ARQ with $3 \mathrm{~mJ} /$ node.

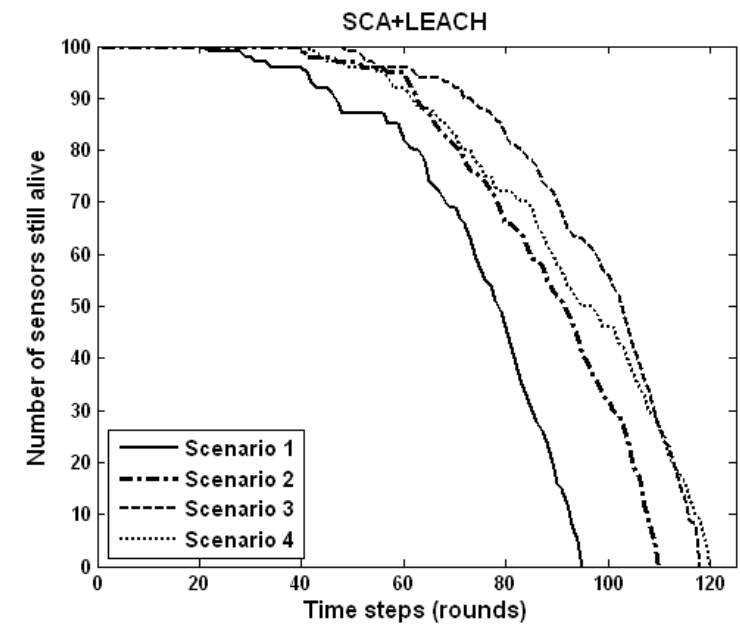

Fig. 5. System lifetime for SCA with LEACH with $3 \mathrm{~mJ} /$ node.

Specific behaviors containing the amount of rounds for the first and last dead node, are shown in Tables II and III.
TABLE II

AMOUNT OF ROUNDS FOR THE FIRST DEAD NODE.

\begin{tabular}{|c|c|c|}
\hline \multirow{2}{*}{ Scenario } & \multicolumn{2}{|c|}{$\begin{array}{c}\text { Rounds for the } \\
\text { first dead node }\end{array}$} \\
\hline & LEACH+T.ARQ & SCA+LEACH \\
\hline One & 28 & 22 \\
\hline Two & 41 & 41 \\
\hline Three & 41 & 49 \\
\hline Four & 42 & 62 \\
\hline
\end{tabular}

TABLE III

AMOUNT OF ROUNDS FOR THE LAST DEAD NODE.

\begin{tabular}{|c|c|c|}
\hline \multirow{2}{*}{ Scenario } & \multicolumn{2}{|c|}{$\begin{array}{l}\text { Rounds for the } \\
\text { last dead node }\end{array}$} \\
\hline \hline & LEACH+T.ARQ & SCA+LEACH \\
\hline One & 82 & 95 \\
\hline Two & 96 & 110 \\
\hline Three & 106 & 118 \\
\hline Four & 120 & 121 \\
\hline
\end{tabular}

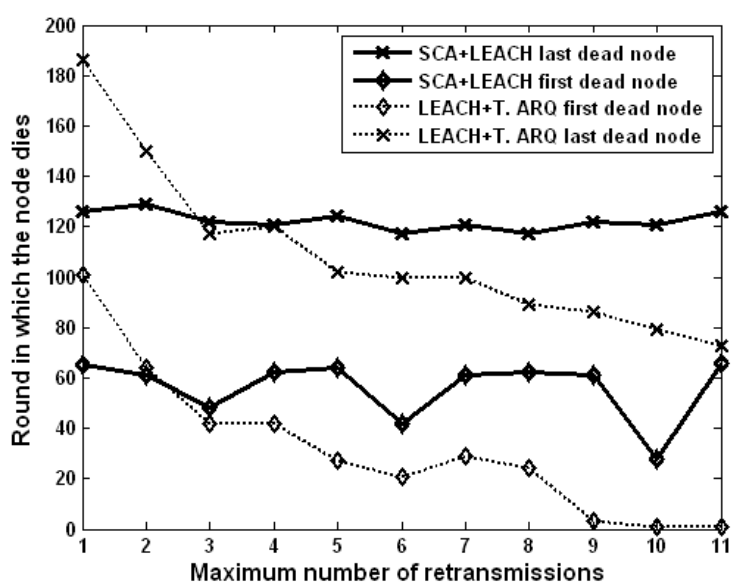

Fig. 6. Lifetime as function of the maximum number of retransmissions.

TABLE IV

OVERALL PACKET LOSS RATE.

\begin{tabular}{|c|c|c|}
\hline \multirow{2}{*}{ Scenario } & \multicolumn{2}{|c|}{$\begin{array}{c}\text { Packet loss } \\
\text { rate }\end{array}$} \\
\hline \hline & LEACH+T.ARQ & SCA+LEACH \\
\hline One & 0.6537 & 0.2105 \\
\hline Two & 0.5042 & 0.1655 \\
\hline Three & 0.4208 & 0.1492 \\
\hline Four & 0.3967 & 0.1537 \\
\hline
\end{tabular}

TABLE V

PACKET LOSS RATE DUE TO NODE DEATH.

\begin{tabular}{|c|c|c|}
\hline \multirow{2}{*}{ Scenario } & \multicolumn{2}{|c|}{$\begin{array}{c}\text { Packet loss rate } \\
\text { due to node death }\end{array}$} \\
\hline \hline & LEACH+T.ARQ & SCA+LEACH \\
\hline One & 0.3657 & 0.9500 \\
\hline Two & 0.3967 & 1.0000 \\
\hline Three & 0.4305 & 1.0000 \\
\hline Four & 0.4076 & 1.0000 \\
\hline
\end{tabular}


The maximum number of retransmissions in this simulation is $N_{r}^{\max }=4$.

The lifetime as a fuction of the maximum number of retransmissions is shown in Fig 6. The round in which the node dies is taken into account, by considering the first and last dead node. Evaluating the first dead node, LEACH with Truncated ARQ scheme is better than SCA with LEACH till $N_{r}^{\max }=2$. Beyond $N_{r}^{\max }=3$, the last system overcomes the former. If the last dead node behavior is observed, LEACH with Truncated ARQ scheme is better than SCA with LEACH till $N_{r}^{\max }=3$. Beyond $N_{r}^{\max }=4$, SCA with LEACH presents a better performance. It is an important result, because it allows the designer of a WSN system to deal with a tradeoff between the lifetime and the maximum number of retransmissions. A high $N_{r}^{\max }$ brings a desirable performance related to the network lifetime parameter (in SCA with LEACH), but also brings, more delay for the transmission system.

The overall packet loss rate (PLR) of the sensor network is given by

$$
\text { PLR }=\frac{\text { Number of lost packets }}{\text { Number of generated packets }}
$$

and it is evaluated in Table IV, as a function of the four propagation contidions. As expected, the packet loss rate decreases as the the channel quality becomes better. For all the scenarios, SCA with LEACH overcomes LEACH with Truncated ARQ scheme. The average packet loss rate for the former system is equal to 0.1697 and for the latter, is equal to 0.4939 , that it is aproximately 3 times bigger. In (16), PLR accounts the losses due to the communication processes (when the maximum number of retransmissions is overcomed) and due to the node death (when a packet is generated by the cluster nodes, but the cluster-head dies). In Table V, the packet loss rate due to the node death is evaluated. SCA with LEACH presents, in scenarios two, three and four, an interesting result that attributes all the losses to the death of the nodes. It assures the eficiency of the proposed communication system, since indicates that when retransmissions are necessary, the packet can be delivered succesfully till the maximum number of retransmissions, indifferent from LEACH Truncated ARQ.

\section{CONCLUSION}

This paper proposed a wireless sensor network system, SCA with LEACH, that combines the use of adaptive cooperative diversity and the LEACH protocol. The system design is based in a clustered architecture and for each round, five clusterheads are elected following the LEACH algorithm. SCA with LEACH assures that cooperative diversity can be invoked only if the sink node receives an erroneous packet from the source cluster-head. Relay cluster-heads are elected based on the results of the truncated ARQ process. The performance analysis was carried out considering a flat Rayleigh fading channel and the diversity coding scheme used the Space-Time Block Codes. The performance of SCA with LEACH system and the LEACH system using the truncated ARQ scheme (LEACH with truncated ARQ) was compared.

Four different SNR ranges were considered in the simulations. As expected, for the best scenarios (higher SNR's), the network lifetime was extended. The simulation results showed that for all the channel scenarios, when the general packet loss rate was evaluated, the SCA with LEACH performance was better and it overcomed approximately 3 times the LEACH with truncated ARQ (in average). The efficiency of the proposed communication was assured, since the measurement of the packet loss rate due to the node death achieved for SCA with $\mathrm{LEACH}$ a result of $100 \%$, for the three latter propagation scenarios.

Two important thresholds were achieved. When the death of the first node was under evaluation, was concluded that till two maximum retransmissions, the lifetime performance was better for LEACH with truncated ARQ. Beyond maximum number of three, the lifetime performance of SCA with LEACH was better. When the last dead node was under evaluation, was concluded that till three maximum retransmissions, the lifetime performance was better for LEACH with truncated ARQ. Beyond maximum number of four, the lifetime performance of SCA with LEACH was better.

In future, the authors intend to enhance the energy conservation scheme and network lifetime, using other protocols like PEGASIS, PEACH, etc.

\section{REFERENCES}

[1] I. F. Akyildiz, W. Su, Y. Sankarasubramaniam, and E. Cayirci. Wireless sensor networks: a survey. Computer Networks, 38:393-422, 2002.

[2] G. Anastasi, M. Conti, M. Francesco, and A. Passarella. Energy conservation in wireless sensor networks: A survey. Ad Hoc Networks, 7(3):537-568, May 2009.

[3] W. R. Heinzelman, A. Chandrakasan, and H. Balakrishnan. Energyefficient communication protocol for wireless microsensor networks. pages 10 pp. vol.2-, Jan. 2000.

[4] V. M. DaSilva and E. S. Sousa. Fading-resistant modulation using several transmitter antennas. IEEE Transactions on Communications, 45(10):1236-1244, Oct 1997.

[5] J. H. Winters, J. Salz, and R. D. Gitlin. The impact of antenna diversity on the capacity of wireless communication systems. IEEE Transactions on Communications, 42(234):1740-1751, Feb/Mar/Apr 1994.

[6] W. T. A. Lopes and M. S. Alencar. Space-time coding performance improvement using a rotated constellation. in Anais do XVIII Simpósio Brasileiro de Telecomunicações (SBrT'2000), Gramado, RS, Brasil, Setembro 2000.

[7] V. Tarokh, H. Jafarkhani, and A. R. Calderbank. Space-time block codes from orthogonal designs. IEEE Trans. Inform. Theory, 45:1456-1467, 1999.

[8] S. M. Alamouti. A simple transmit diversity technique for wireless communications. IEEE Journal on Selected Areas in Communications, 16(8):1451-1458, 1998.

[9] H. Jafarkhani. Space-time coding. Theory and Practice. Cambridge University Press, 2005.

[10] E. Malkamaki and H. Leib. Performance of truncated type-II hybrid ARQ schemes with noisy feedback over block fading channels. IEEE Transactions on Communications, 48(9):1477-1487, Sep 2000.

[11] Q. Liu, S. Zhou, and G. B. Giannakis. Cross-Layer combining of adaptive Modulation and coding with truncated ARQ over wireless links. IEEE Transactions on Wireless Communications, 3(5):1746-1755, Sept. 2004.

[12] F. Xiangning and S. Yulin. Improvement on leach protocol of wireless sensor network. pages 260-264, Oct. 2007.

[13] Lin Dai and K. Letaief. Throughput maximization of ad-hoc wireless networks using adaptive cooperative diversity and truncated ARQ. IEEE Transactions on Communications, 56(11):1907-1918, November 2008.

[14] B. Vucetic and J. Yuan. Space-time Coding. John Wiley \& Sons, 2003.

[15] Y. Chen and Q. Zhao. On the lifetime of wireless sensor networks. IEEE Commun. Lett., 9(11):976-978, November 2005. 